\title{
Acoustophoretic volumetric displays using a fast-moving levitated particle
}

Cite as: Appl. Phys. Lett. 115, 064101 (2019); https://doi.org/10.1063/1.5113467

Submitted: 04 June 2019. Accepted: 09 July 2019 . Published Online: 05 August 2019

Tatsuki Fushimi (iD), Asier Marzo (D), Bruce W. Drinkwater (D), and Thomas L. Hill (D)
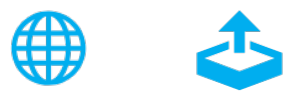

View Online

Export Citation

\section{ARTICLES YOU MAY BE INTERESTED IN}

TinyLev: A multi-emitter single-axis acoustic levitator

Review of Scientific Instruments 88, 085105 (2017); https://doi.org/10.1063/1.4989995

Phase and amplitude modulation with acoustic holograms

Applied Physics Letters 115, 053701 (2019); https://doi.org/10.1063/1.5110673

Interlayer modulation on the dynamic magnetic properties of $\mathrm{L}_{0} \mathrm{O}-\mathrm{FePt} / \mathrm{NM} /[\mathrm{CoNi}]_{5}$ composite film structures

Applied Physics Letters 115, 062401 (2019); https://doi.org/10.1063/1.5099182

Lock-in Amplifiers Find out more today

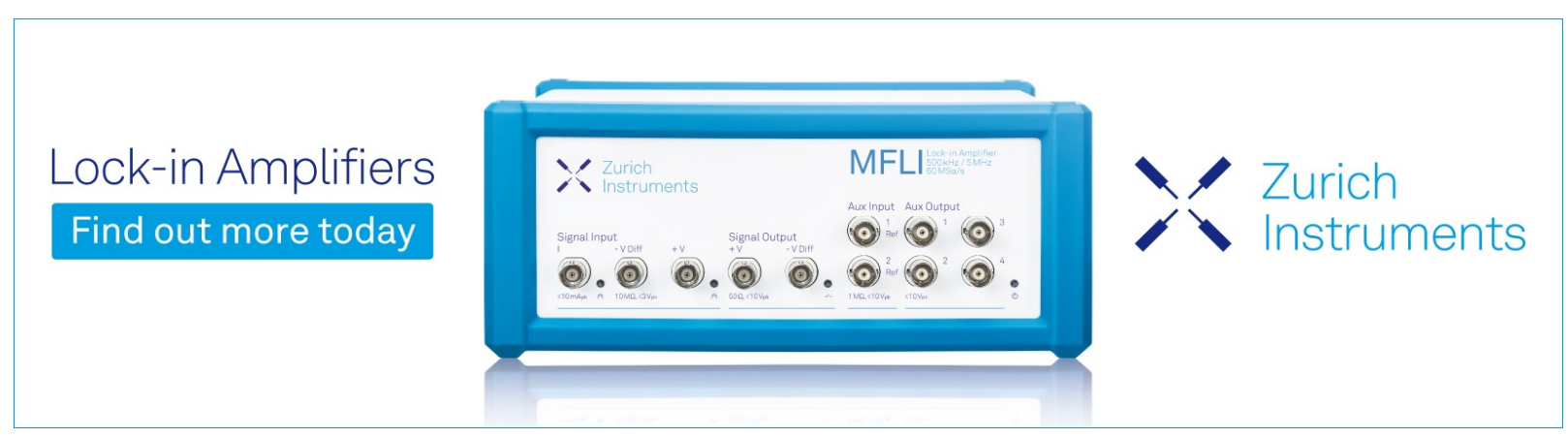




\title{
Acoustophoretic volumetric displays using a fast-moving levitated particle
}

\author{
Cite as: Appl. Phys. Lett. 115, 064101 (2019); doi: 10.1063/1.5113467 \\ Submitted: 4 June 2019 . Accepted: 9 July 2019 . \\ Published Online: 5 August 2019
}

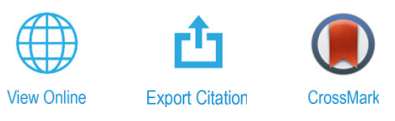

Tatsuki Fushimi, ${ }^{1, a)}$ (D) Asier Marzo, ${ }^{2}$ (D) Bruce W. Drinkwater, ${ }^{1}$ (D) and Thomas L. Hill' (D)

\author{
AFFILIATIONS \\ 'Department of Mechanical Engineering, University of Bristol, BS8 1TR Bristol, United Kingdom \\ ${ }^{2}$ UpnaLab, Universidad Pública de Navarra, Pamplona 31006, Navarre, Spain

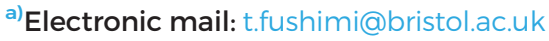

\begin{abstract}
Displays have revolutionized the way we work and learn, and thus, the development of display technologies is of paramount importance. The possibility of a free-space display in which $3 \mathrm{D}$ graphics can be viewed from $360^{\circ}$ without obstructions is an active area of research-holograms or lightfield displays can realize such a display, but they suffer from clipping and a limited field of view. Here, we use a phased array of ultrasonic emitters to realize a volumetric acoustophoretic display in which a millimetric particle is held in midair using acoustic radiation forces and moved rapidly along a 3D path. Synchronously, a light source illuminates the particle with the target color at each $3 \mathrm{D}$ position. We show that it is possible to render simple figures in real time (10 frames per second) as well as raster images at a lower frame rate. Additionally, we explore the dynamics of a fast-moving particle inside a phased-array levitator and identify potential sources of degradation in image quality. The dynamics are nonlinear and lead to distortion in the displayed images, and this distortion increases with drawing speed. The created acoustophoretic display shows promise as a future form of display technology.
\end{abstract}

Published under license by AIP Publishing. https://doi.org/10.1063/1.5113467

A volumetric display is a device that "permits the generation, absorption, or scattering of visible radiation from a set of localized and specified regions within a physical volume." $\mathrm{A}$ "free-space display" is a type of volumetric display that operates in midair and does not have barriers between the user and the images.

Recently, Smalley et al. presented the concept of a photophoretictrap based volumetric display (OTD: Optical Trap Display). ${ }^{3}$ An OTD has significant advantages compared to holograms or lightfield displays since it renders images that can be observed from different angles without clipping. ${ }^{3}$ In the OTD, a micrometric particle is held in midair and displaced, at high speed (maximum $1.8 \mathrm{~m} \mathrm{~s}^{-1}$ ), by an invisible photophoretic trap, induced with a laser. ${ }^{3}$ The trap translates the particle that is illuminated at the same time by a visible light source and rapidly traces the desired path inside the working space, thus scattering light from different positions to form a visible afterimage. This image can be directly observed without wearing any device and from different perspectives. Hence, this demonstrated that photophoretictrap based systems can render precise images of the centimeter scale in midair. However, the OTD has significant disadvantages, such as requiring high-power lasers and providing a limited display volume. Electrostatic-trap based volumetric displays were demonstrated by Berthelot and Bonod. ${ }^{4}$ In this concept, a micrometric charged particle was levitated and moved by a set of electrodes with varying electrostatic charges. This electrostatic trap is not as hazardous as a laser system, but the rendered images were on the micrometer scale.

Here, we present the concept of a volumetric acoustophoretic display. Similar to electrostatic ${ }^{4}$ and photophoretic ${ }^{2}$ volumetric displays, we rapidly displace a levitated particle while illuminating it to create 3D shapes. However, we achieve this using acoustic radiation forces (i.e., acoustophoresis) to hold and move the particle. Acoustophoretic traps in the high-ultrasonic range are thought to be safe for humans ${ }^{5}$ and simpler (i.e., a simple acoustic levitator can be manufactured from low-cost off-the-shelf components and used at home ${ }^{6,7}$ ), and its region of operation can be as large as half a meter.

A number of authors have previously attempted to create a freespace display using acoustophoresis. Ochiai et al. showed crosses and uniform 2D grids made out of levitating particles. ${ }^{8}$ Sahoo et al. presented a screen made of levitated dielectric particles that can be flipped individually using an electric field. ${ }^{10}$ Uno et al. demonstrated the levitation of an LED to draw images in air using long exposure camera shots. ${ }^{11}$ Marzo and Drinkwater controlled multiple particles (up to 25) independently to create different $3 \mathrm{D}$ shapes. ${ }^{12}$ However, these approaches formed the $3 \mathrm{D}$ objects to be displayed at each instant of time using multiple levitated particles. This leads to coarse objects 
being shown due to the limited number of particles. Additionally, those displays were monochromatic. Here, we use a single rapidly moving particle that traces $3 \mathrm{D}$ paths, while being illuminated, and exploits the persistence of vision to render free-space 3D graphics. We present the hardware used in a prototype and assess its performance limits.

Figure 1(a) shows an overview of the acoustophoretic-trap freespace volumetric display. The image is constructed by a series of voxels that are created when the particle reaches a particular position at a given time and is illuminated by an RGB (red, green, and blue) LED (light-emitting diode). Therefore, a 3D graphic is represented as a sequence of particle positions and RGB values. The colors are represented as an 8-bit signal for each RGB channel and the particle position as $(x, y, z)$ at a given time, $t$. The colors are sent to the RGB LED, and the emission phases for a given trapping position are calculated and sent to an array of ultrasonic transducers. The array-based ultrasonic levitator device is controlled by an FPGA (field-programmable gate array) board (ALTERA CoreEp4CE6) which is connected to a PC via UART (universal asynchronous receiver-transmitter) serial communication (1.5 Mbauds) and produces 60 channels of square-waves each controllable with a $\pi / 64$ rad phase resolution. ${ }^{6}$ The wave is transmitted to MOSFET drivers (TC4427a) which amplify the FPGA signal to a voltage provided by an external DC power supply (RS Pro IPS 303DD). These amplified signals are sent to 60 ultrasonic transducers (Murata MA40S4S) arranged as two opposed arrays, each of 30 transducers. Signals are generated to focus the array at the trap position (see the supplementary material), and this creates an antinode at the focal point with one node above and another below where a small Expanded Polystyrene (EPS) particle with a diameter of $\approx 1.5 \mathrm{~mm}$ can be trapped. A different size of particle may be utilized for the volumetric display; however, for larger particles $(>1.5 \mathrm{~mm})$, the small particle assumptions in the Gor'kov model become invalid and the full model shows that the force per unit volume decreases. Smaller particles are more susceptible to air currents as the drag force becomes increasingly significant [drag scales with area and acoustic radiation force (Gor'kov) scales with volume] and particles $<0.5 \mathrm{~mm}$ are often swept out of the traps. ${ }^{13}$ The working volume of the display is approximately the size of the employed single-axis acoustic levitator $\left(4 \times 5 \times 8 \mathrm{~cm}^{3}\right)$. The RGB LED is located on the side of the acoustic levitator. The LED illumination will scatter off the particle and give color to each voxel of the $3 \mathrm{D}$ path graphics. When the particle moves sufficiently fast while it is being illuminated, it generates a continuous $3 \mathrm{D}$ path due to the

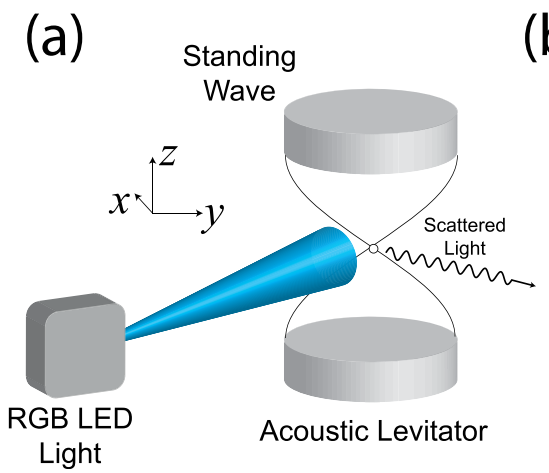

(b)

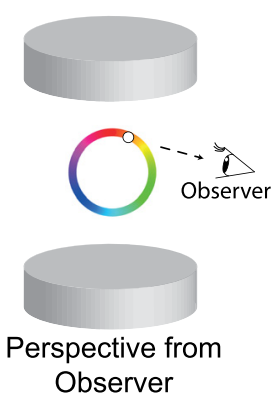

FIG. 1. Overview of acoustophoretic volumetric display. (a) Schematic diagram of the experimental setup. (b) Perspective from the observer. persistence of the vision effect, as shown in Fig. 1(b). In this implementation, one RGB LED has been utilized for the illumination; the visibility from a full $360^{\circ}$ can be ensured by increasing the number of light sources around the levitator (see the supplementary material for the light intensity measurement around the levitator). Advanced illumination techniques, such as a light source that accurately tracks the position of the particle, may be employed to further improve the contrast and clarity of the displayed image.

A series of experiments were conducted to identify potential causes of image degradation and show paths for achieving accurate rendering in a real-time acoustophoretic volumetric display. In addition to the apparatus shown in Fig. 1(a), an LED source with a diffuser and a camera (CM3-U3-13Y3M-CS) were used to accurately track the particle for display calibration, as shown in the supplementary material.

First, the static equilibrium points of the particle in the acoustic levitator were measured experimentally, while moving the focus of the levitator along a 2D grid pattern with a particle trapped at the focus. For each focal position, the particle was allowed to rest (for $1.25 \mathrm{~s}$ ) and reach its equilibrium point. In theory, the equilibrium point should exactly match the focal point, with a small offset on the vertical direction due to the gravitational forces. However, recent studies ${ }^{9,14,15}$ have shown that, in practice, the equilibrium points of focused acoustic levitators deviate from the ideal equilibrium points (typical magnitude of deviation $\approx 0.5 \mathrm{~mm}$; an example of a deviation map is shown in the supplementary material). The exact cause of these deviations has not yet been identified and remains an open problem. Previous studies suggest scattering from the transducer surface, temperature variations, inaccuracies in the positions of the transducers, and manufacturing variations between the elements as potential contributing factors. This deviation can significantly affect the accuracy of the particle position and thus the quality of the rendered graphics.

Consequently, we characterized the deviation at each point and used these values to update the focal points. ${ }^{15}$ This static calibration method allows the acoustophoretic display to rapidly generate various raster graphics as shown in Figs. 2(a)-2(f). Figure 2(a) shows the rendered University of Bristol logo, and Fig. 2(b) is a close up from the front. Figure 2(c) shows an example of an image of a stop sign. Figures 2(d)-2(f) show a 3D wireframe airplane observed from multiple angles (see the supplementary material for the 3D static calibration
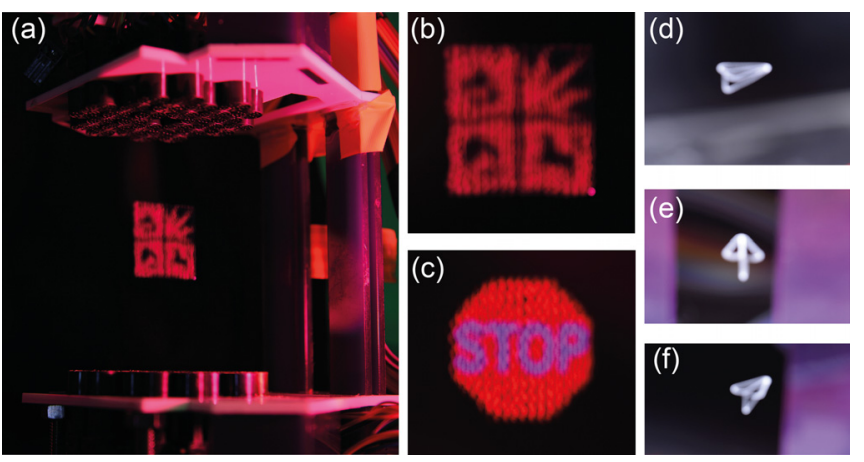

FIG. 2. Examples of images created with the acoustophoretic display device. (a) Image together with the acoustic levitator render (draw) time $\left(T_{r}=20 \mathrm{~s}\right)$, (b) image in (a) taken from the front, (c) an example of an image of a stop sign $\left(T_{r}=20 \mathrm{~s}\right)$, and $(\mathrm{d})-(\mathrm{f}) 3 \mathrm{D}$ wireframe volumetric image of an airplane viewed from various angles $\left(T_{r}=5 \mathrm{~s}\right)$. 
method). With the current prototype, the draw time is $20 \mathrm{~s}$ for Figs. $2(\mathrm{a})-2(\mathrm{c})$ and $5 \mathrm{~s}$ for Figs. 2(d) $-2(\mathrm{f})$, and we note that this rendering capability is identical to that of the latest photophoretic display where a similarly scaled image was rendered at an exposure time of 8-60 s. ${ }^{2}$

Increasing the velocity of the particle distorts the shape of the volumetric image and, ultimately, the particle is ejected from the acoustic trap. This effect can be observed in the images formed by simpler trajectories as shown in Figs. 3(a)-3(c) where the velocity of the particle is increased so that the images are formed at 1,5 , and $10 \mathrm{~Hz}$ [Fig. 3(a) is the physical implementation of Fig. 1(b), and the video of vector graphic rendering is available as a supplementary material video]. At the lowest velocity $(1 \mathrm{~Hz})$, the target shapes are formed as specified via static calibration; however, as the velocity increases, the image gets deformed. Rendering frequencies of at least $10 \mathrm{~Hz}$ are necessary to draw an image to the human eye in real time. While our prototype display can achieve these frequencies, currently, this is only possible for small and simple shapes in the size of 5-6 mm.

Although this static calibration significantly increases the image quality, ${ }^{15}$ the results in Fig. 3 demonstrate that this calibration decreases in effectiveness as the speed of the particle increases. Dynamic optimization of the trajectory is inherently difficult due to the nonlinear dynamics of particles inside an acoustic levitator. ${ }^{16}$ To characterize the dynamics of the particle and the fidelity of the displacement, the focal point was oscillated vertically along the $z$-axis with varying amplitudes in the range of $0.5 \mathrm{~mm} \leq A \leq 10 \mathrm{~mm}$ with an increment of $0.5 \mathrm{~mm}$ and a frequency ranging from 2 to $35 \mathrm{~Hz}$ with increments of $1.0 \mathrm{~Hz}$. The oscillation frequency of the particle was increased gradually before maintaining a constant frequency for $10 \mathrm{~s}$ to take steady-state measurements. At each frequency step, the particle oscillation was recorded at 150 FPS using the same experimental setup as for the static equilibrium point experiment. The footage of the particle movement was analyzed using MATLAB Image Processing

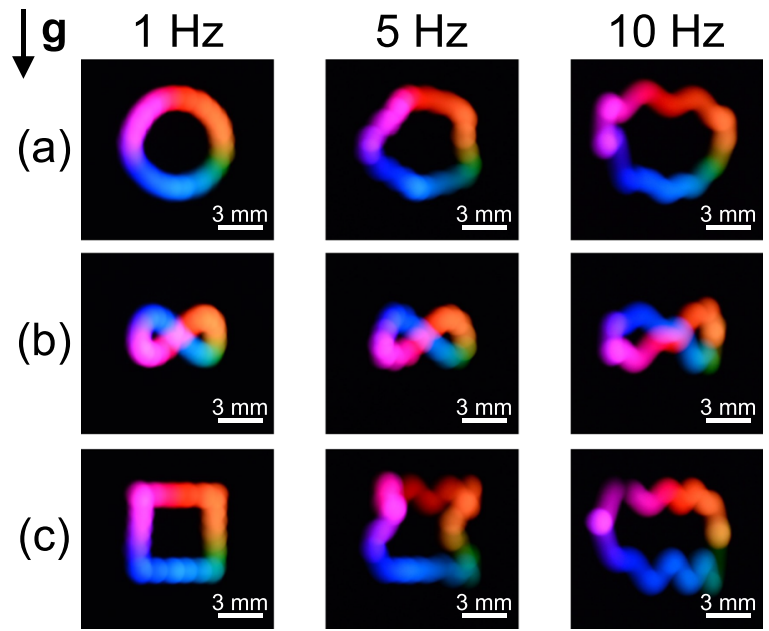

FIG. 3. Rendered vector graphics. (a) Circle with a diameter of $6 \mathrm{~mm}$, (b) infinity symbol with a horizontal length of $5 \mathrm{~mm}$, and (c) square with a side length of $5 \mathrm{~mm}$. The rendering (drawing) frequency changes from 1,5 , to $10 \mathrm{~Hz}$, and the exposure time of each instance is equal to the rendering frequency. The oscillation in the picture involves slow oscillation (from the trajectory) and fast oscillation (the particle oscillating around the acoustic trap).
Toolbox, and the particle's center coordinates were extracted using the Hough Circle Transform. The amplitude and frequency were then extracted in postprocessing (detailed method of processing of data is available in the supplementary material), and the experimental results are shown in Figs. 4(a)-4(c), represented in terms of the Z- and Y-axis amplitude error and $Y$-axis frequency ratio $\left(F_{R}=f_{P} / f_{z}\right.$, where $f_{P}$ is the principal frequency of oscillation in the Y-direction, identified via DFT (Discrete Fourier Transform) analysis, and $f_{z}$ is the input $z$ axis oscillation frequency). The amplitude error is the absolute difference between the oscillation amplitude of the trap and the response amplitude. If a dynamic model is formulated without considering the static deviation, off-axis movement, as shown in Fig. 4(b), will not be predicted (see the supplementary material). Qualitative prediction of the off-axis movement was possible [see Figs. 4(d)-4(f)] using a hybrid model which included the experimentally observed static deviation. This hybrid model firstly uses Huygens' principle to predict the acoustic field and then Gor'kov's method ${ }^{17}$ to extract the forces acting on a small particle (see the supplementary material for further details). Next, it is assumed that the force profile remains unchanged (relative to step 1) but that it was shifted spatially by a certain amount. This shift is obtained from the difference between the calibration maps and the numerical prediction of static equilibrium points. This difference was then fitted into the polynomial approximation of the Gor'kov potential and generated a hybrid model (see the supplementary material for details). The gaps in the numerical simulation [Figs. 4(d)-4(f)] are regions where there is significant particle oscillation, in either axis, that it is out of the region where the numerical model is valid $( \pm 2 \mathrm{~mm}$ in the $y$ axis and $\pm 3.5 \mathrm{~mm}$ in the $z$ axis). There exist some discrepancies between the experimental and simulated responses for the peaks (i.e., resonances) in the off-axis movement, as shown in Figs. 4(b) and 4(e). These differences may be caused by error in the model of the acoustic radiation force profile away from the static equilibrium, which was not calibrated in the hybrid model (i.e., the hybrid model only corrects for the static deviation). However, the good qualitative and reasonable quantitative agreement suggests that this model is able to explain the causes of the dynamic observations, such as undesirable off-axis movement, which will inevitably decrease the image quality in the volumetric display. Moreover, the hybrid model simplifies the process of identifying the relevant dynamics, in comparison to the development of a forward model involving, e.g., scattering from each transducer or nearby surfaces, which is computationally more expensive and challenging to implement. The dynamic response for each trajectory will differ with the geometry and output amplitude of the levitator, and the result for the $\mathrm{Z}$ (vertical) oscillation does not superimpose with the response for the different shapes in Fig. 3.

One key observation from Fig. 4 is that the cut off region (i.e., the frequency and amplitude combinations that result in the particle ejection from the trap) of the results in (a)-(c) occurs earlier, in comparison with the simulated response in (d)-(f). In the experiment, the particle was oscillated until it was ejected from the trap, and this early cut off signifies that the operational region of the levitator is significantly smaller than expected, compared to the numerical predictions (reproducibility of the ejection point is discussed in the supplementary material). We also note that doubling the operational voltage from $9 \mathrm{~V}$ to $18 \mathrm{~V}$ does not improve the levitator's performance (see the supplementary material). From these observations, we attribute our current performance limit to the narrow bandwidth of the transducers, which 

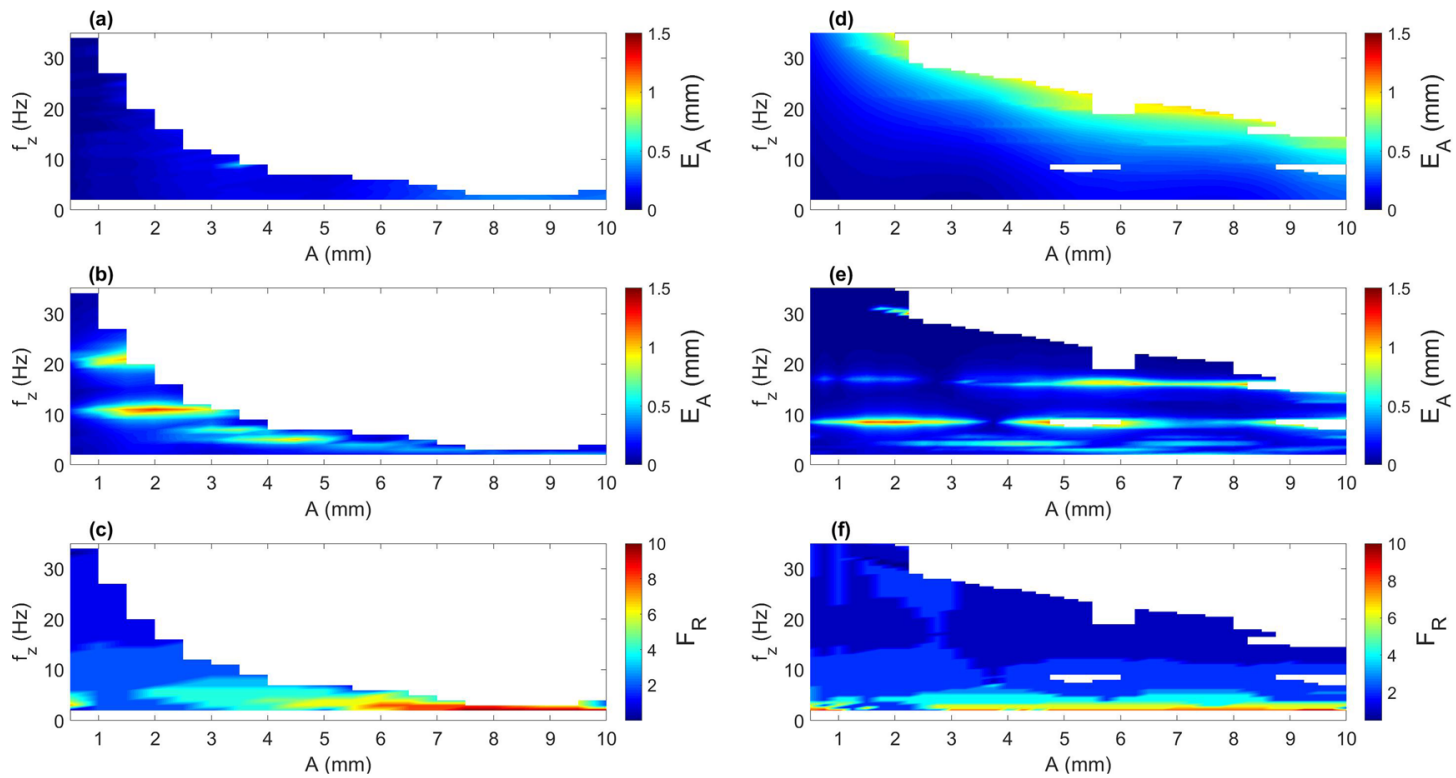

FIG. 4. Comparison of experimental and simulated responses to a vertical focal point oscillation of varying amplitudes and frequencies. (a) shows the experimental Z-axis error, in terms of the absolute difference between the oscillation amplitude of the trap and response amplitude of the particle $\left(E_{A}\right)$, (b) shows the experimental $Y$-axis oscillation, $(c)$ shows the ratio between the $Y$-axis response frequency and the $Z$-axis input frequency $\left(F_{R}\right)$, and $(d)-(f)$ show the numerically simulated $Z$-axis error, $Y$-axis error, and $Y$-axis frequency ratio using the hybrid model, respectively.

leads to a reduced ability to rapidly change the phase of the ultrasonic signal. As the perturbation frequency and amplitude increase for the focal point, the transducer phases need to vary more rapidly and result in a reduced emitted ultrasonic signal (see the supplementary material). The effect on the acoustic radiation force is difficult to assess due to the time-varying pressure field. In the supplementary material, we model the dynamic response of the narrow band transducer as a single degree of freedom system with empirically derived parameters. With this model, we show that the current transducers are limiting as they are unable to respond to the required rapid phase variations. Furthermore, as described before, an increase in voltage did not change the point at which ejection occurred, a finding that is in agreement with the hypothesis that the transducer dynamics are the limiting factor. We further hypothesize (see the supplementary material) that transducers that are capable of outputting similar pressure levels over a wider bandwidth can overcome this limit. Beyond the transducer limit, Fig. 4 suggests that the resonance effects caused by the trapping dynamics can cause particles to be ejected from the acoustic trap.

We note that the maximum velocity recorded on this levitator is $0.67 \mathrm{~m} \mathrm{~s}^{-1}$ which is comparable to that achieved in the other acoustic systems, e.g., $0.25 \mathrm{~m} \mathrm{~s}^{-1}$ in Marzo et al. ${ }^{18}$ However, the maximum achievable speed varies with the rendered shape and it is the particle acceleration (which sets the required force) that is the limiting factor and the subject of this paper.

We have presented the concept of an acoustophoretic volumetric display in which dynamic acoustic fields are used to rapidly move a levitating particle that traces different $3 \mathrm{D}$ graphics. The movement is coordinated with direct illumination of the particle and, due to the persistence of vision effect, the user perceives a free-form floating graphic. It has similar performance to the latest free-space volumetric display using optical traps, ${ }^{2}$ but it is safe to be used outside of the laboratory. A prototype was developed and evaluated in terms of speed and accuracy. A hybrid model showed that the current performance limits were due, in part, to the deviation of the static particle equilibrium points from their expected values. Our implementation was subject to a further limitation due to the narrow bandwidth of the emitting transducers. Despite this limitation, we created a volumetric image of a $5-6 \mathrm{~mm}$ sized circle, infinity sign, and a square that were observable in real time $\left(T_{r}=0.1 \mathrm{~s}\right)$. We also predict that better performing (i.e., wider bandwidth) transducers would permit the rendering of raster screens, given the same hardware. Hence, this acoustophoretic approach has the potential to become a future form of display technology.

See the supplementary material for the details on the experimental setup, calculation of phase delays and equilibrium points, static calibration method, recording method for the volumetric images, dynamic response data processing and prediction method, hybrid model formulation, light intensity measurement, reproducibility study of ejection frequency, observation of transducer off resonance, transducer modeling, and effects of the wider bandwidth transducer. A supplementary video of vector graphics is also available.

T.F. was funded through the Japan Student Services Organization (JASSO) Student Exchange Support Program (Graduate Scholarship for Degree Seeking Students). This project was funded by the UK Engineering and Physical Science Research Council (No. EP/N014197/1). This work was carried out using the computational facilities of the Advanced Computing Research Centre, University of Bristol-http://www.bris.ac.uk/acrc/. We would like to thank Mr. Stephen Isles for manufacturing 
components for the experimental setup. Sufficient information is available in this manuscript and the supplementary material to recreate the numerical and experimental results. The data presented in this work are openly available at Zenodo at https://doi.org/ 10.5281/zenodo.3299281.

\section{REFERENCES}

'B. G. Blundell and A. J. Schwarz, Volumetric Three-Dimensional Display Systems (John Wiley \& Sons, Incorporated, 2000).

${ }^{2}$ D. Smalley, T. Poon, H. Gao, J. Kvavle, and K. Qaderi, Opt. Photonics News 29, 26 (2018).

${ }^{3}$ D. E. Smalley et al., Nature 553, 486 (2018).

${ }^{4}$ J. Berthelot and N. Bonod, Opt. Lett. 44, 1476 (2019).

${ }^{5}$ T. G. Leighton, Proc. R. Soc. A 472, 20150624 (2016).

${ }^{6}$ A. Marzo, T. Corkett, and B. W. Drinkwater, IEEE Trans. Ultrason., Ferroelectr., Frequency Control 65, 102 (2018).

${ }^{7}$ A. Marzo, A. Barnes, and B. W. Drinkwater, Rev. Sci. Instrum. 88, 085105 (2017).

${ }^{8}$ Y. Ochiai, T. Hoshi, and J. Rekimoto, PLoS One 9 , e102525 (2014).
${ }^{9}$ S. Inoue, S. Mogami, T. Ichiyama, A. Noda, Y. Makino, and H. Shinoda, J. Acoust. Soc. Am. 145, 328 (2019).

${ }^{10}$ D. R. Sahoo, T. Nakamura, A. Marzo, T. Omirou, M. Asakawa, and S. Subramanian, in Proceedings of the 29th Annual Symposium on User Interface Software and Technology-UIST '16 (ACM Press, New York, New York, USA, 2016), pp. 437-448.

"Y. Uno, H. Qiu, T. Sai, S. Iguchi, Y. Mizutani, T. Hoshi, Y. Kawahara, Y. Kakehi, and M. Takamiya, Proc. ACM Interact., Mobile, Wearable Ubiquitous Technol. 1, 166 (2017).

${ }^{12}$ A. Marzo and B. W. Drinkwater, Proc. Natl. Acad. Sci. 116, 84 (2019).

${ }^{13}$ L. Cox, A. Croxford, B. W. Drinkwater, and A. Marzo, Appl. Phys. Lett. 113, 054101 (2018).

${ }^{14} \mathrm{M}$. Prisbrey and B. Raeymaekers, Phys. Rev. Appl. 10, 034066 (2018).

${ }^{15}$ T. Fushimi, A. Marzo, T. L. Hill, and B. W. Drinkwater, in 2018 IEEE International Ultrasonics Symposium (IUS) (2018), p. 1.

${ }^{16}$ T. Fushimi, T. L. Hill, A. Marzo, and B. W. Drinkwater, Appl. Phys. Lett. 113, 034102 (2018).

${ }^{17}$ L. P. Gor'kov, Sov. Phys. Dokl. 6, 773 (1962).

${ }^{18}$ A. Marzo, S. A. Seah, B. W. Drinkwater, D. R. Sahoo, B. Long, and S. Subramanian, Nat. Commun. 6, 8661 (2015). 